\title{
LETTER
}

\section{Delivering food safety}

\author{
Kaye BASFORD (凶), Richard BENNETT, Joanne DALY, Mary Ann AUGUSTIN, Snow BARLOW, \\ Tony GREGSON, Alice LEE, Deli CHEN, Matt WENHAM
}

Australian Academy of Technological Sciences and Engineering, GPO Box 4055 Melbourne VIC 3001, Australia

\begin{abstract}
A delegation from the Australian Academy of Technological Sciences and Engineering traveled to Beijing in April 2016 to jointly run a workshop on technology advances in food safety with the Chinese Academy of Engineering. This brief summary from the Australian delegation identifies the pyramid of interlocking issues which must be addressed to deliver food safety. Systems and technology provide the necessary base, on which culture and then trust can be built to facilitate the delivery of food safety now and in the future.
\end{abstract}

Keywords culture, food safety, systems, technology, trust

\section{Introduction}

A workshop was jointly run in Beijing in April 2016 by the Chinese Academy of Engineering (CAE) and the Australian Academy of Technological Sciences and Engineering (ATSE) on technology advances in food safety. This brief article gives a summary report from the Australian delegation.

We agreed that we live in a world with:

- increasing population and urbanization,

- changing agricultural practices and climate,

- increasing technological intervention in food production,

- increasing volume and diversity of trade in food,

- changing requirements for food preparation,

- increasing numbers of socially connected consumers, and

- increasing demand for safe food.

To deliver food safety, we identified a pyramid of interlocking issues - systems and technology are essential

Received September 21, 2016; accepted December 12, 2016

Correspondence: k.e.basford@uq.edu.au for a solid base, while culture and trust build on these (Fig. 1).

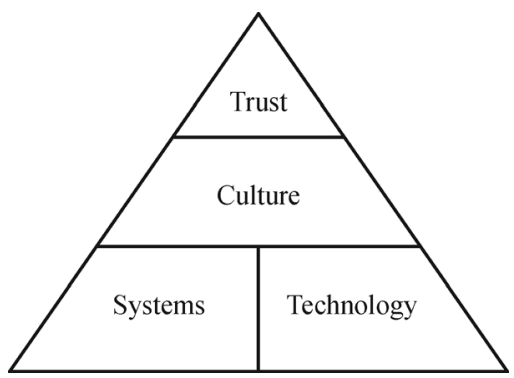

Fig. 1 Pyramid of interlocking issues to deliver food safety

\section{Systems and technology}

We need to ensure food safety along the whole agrifood chain, or rather web, as we now have complex meal solutions involving both fresh and processed food (of domestic and international origin). It is quite possible that the average two-course meal served in a western style restaurant or purchased from a supermarket retailer has ingredients from a number of countries. Consumers expect food that is $100 \%$ safe and their zero tolerance of anything less requires the joint efforts of all stakeholders, and effective coordination of government policy and regulation with industry control and management.

Governments develop standards to protect their population from illness and injury associated with unsafe food. While these standards often vary in detail from country to country, they are almost universally based on the Codex HACCP program of identifying food safety hazards and implementing appropriate controls at stages of the supply chain that can effectively minimize or eliminate those hazards. Government standards also provide compliance points for regulated agrochemical residues and microbiological limits, and provide a mechanism for applying penalties for noncompliance. Monitoring and enforcement requires resources in the form of competent auditors and an 
effective audit management system.

While regulation provides an overarching food safety structure, commercial organizations leverage this structure in order to meet additional, internal requirements. While noncompliance may bring about regulated penalties, commercial enterprises may be more greatly impacted by ongoing reputational harm as a consequence of an outbreak of foodborne illness attributed to their products. This harm can apply whether the organization is a food manufacturer or retailer, and whether they were the source of the outbreak or not.

As foodborne illness reporting has been enhanced and consumers have become more likely to communicate the supposed cause of their illness via social media, food safety management needs to be much more rigorous than ever to prevent negative economic impacts and should take a whole-of-chain adoption approach. Over time, commercial organizations have developed or adopted proprietary food safety standards that meet both regulatory and commercial needs, to the point where there is now a plethora of standards available. This has resulted in many vendor businesses being required to meet multiple but often very similar standards in order to supply multiple customers, with a commensurate increase in direct and indirect costs that can be difficult to justify on the basis of food safety outcomes.

Fortunately, we appear to have passed the peak in proliferation of standards with the evolution of standard equivalence schemes. Existing schemes can be benchmarked against global best practice in food safety management. Customer groups such as retailers, wholesalers and processors can opt to accept vendors certified to any of a number of schemes, knowing that each scheme is equivalent to a global benchmark for best practice. The Global Food Safety Initiative is the predominant example of food safety equivalence and is gradually increasing the number of schemes under its umbrella.

As fewer obstacles to implementation of food safety schemes give better results, there is a need for straightforward guidelines and risk assessment tools. While standards may be generic and universal, specific differences associated with crops, climates and countries can be addressed by specific guidelines. This type of guidance enables growers to successfully tailor their documentation to meet the requirements of global standards and their own particular risk profile. In particular, the need to address risks, such as specific sites, water quality, specific contaminants, particular harvest practices and other unique inputs or activities, can be accommodated.

Technology and engineering involved in food production, processing and preservation has enabled us to remove impurities, such as pollutants and residues, while not adding adverse toxins. Food processing has an important role in food and nutrition security. Both conventional (e.g., heat treatment, drying) and emerging (e.g., high pressure, ultrasound) food processing technologies may be used for the conversion of agricultural produce to safe, healthy, convenient and shelf-stable foods that are acceptable to the consumer. Food processing technologies will be needed to complement other strategies that will enable the reduction of edible food waste. Ethical application of food processing for production of safe foods and ingredients that meet specifications of composition and quality will help engender the trust of consumers.

The Internet and global information systems (such as the Internet of things) enable improved growth and harvesting processes (through monitoring and control) while collecting data that will lead to productivity and safety improvements. Technology will play an even more important role in the seamless integration of product identification and global traceability through information collection (barcode and sensor technology), transmission (data exchangeability through common formats and interface standards), and processing (data mining and food quality diagnosis). Real-time access to documents and records at all stages will be commonplace, and will require advanced information and communication technology for data security.

\section{Culture}

While systems and technology are the tools by which food safety standards can manage food safety, the best systems and technology will fail if the workforce culture is not orientated toward producing safe food $24 \mathrm{~h}$ per day, 365 days per year. This culture needs to be led by example from the top of the organization, be that a family business or a multinational, and find its way throughout the organization to the most junior and transient employees. This culture can be difficult to achieve. For example, some farmers may not see themselves as an intrinsic player in the food chain, instead seeing themselves as a provider of raw materials to food businesses. Nevertheless, problems that occur along the supply chain often start with the producer.

We all want a culture in which producers and agribusinesses along the value chain take responsibility for food safety and strive for global best practice. This approach requires that all participants in food production and processing work proactively, rather than reactively, to identify risks. There is a need to employ a risk analysis framework involving science-based assessment, policybased management, and open communication. Unannounced audits (requiring real-time data) will become commonplace and welcome, while better labeling will meet consumer demands for transparency and sustainability.

Again, this all begins with the producers who understand the importance of farm and produce hygiene and safety for multiple customers in domestic and international markets. 
Such producers are proactive with safety and quality procedures to create provenance - they generate farm produce in a safe and environmentally friendly manner, adhering to all codes of practice and relevant legislation. They can be proud of their clean and green image, while simultaneously increasing productivity and profitability and preserving ecosystem health.

However, not all consumers are driven by the same issues with many people making trade-offs among price, convenience and attributes. People do not always do what they say - they may indicate their desire to buy local food, yet will buy on price in the supermarket. Although food has never been safer, some consumers are anxious about safety and become sensitized to different products by apparent mismanagement of major food scandals, such as bovine spongiform encephalopathy in meat. In response, some consumers turn to perceived proxies for food safety such as natural, organic or local food.

Public perception of risks in food safety is not the same as actual or technological risks. Attitudes to food safety are often influenced by values, rather than by logic and technical information. This is particularly so when information is complex with people tending to make emotional judgments. Messages that do not align with values tend to be rejected or dismissed; and attitudes not formed by logic or facts are not influenced by logical or factual arguments. In this context, new technology is seen to be potentially risky and needs to demonstrate considerable benefits to consumers to be acceptable. Once the perception of unsafe or undesirable has been published, it is very hard to reverse, so in addition to benefits to consumers, perceived risks must be managed very carefully.

Behaviors and attitudes of people toward a hazard are driven more by their perception of risk, rather than the estimates of actual or technological risk (such as morbidity and mortality rates) provided by experts. Consumers worry more about these technological risks than they do about issues over which they have more control. Microbial contamination is the greatest risk in food safety, but people worry about other contaminants added during production or manufacture. For example in a UK study ${ }^{[1]}, 15 \%$ of people worried strongly about hygiene in home preparation of food compared with over $50 \%$ who were concerned about hormones, antibiotics and pesticides in food.

Much attention in food has focused on genetically modified (GM) crops. However, little is known about the degree to which responses to GM food reflects attitudes to human intervention in modern food production more generally. Recent research in Australia ${ }^{[2]}$ has shown that concerns about GM is a specific example of concern about food integrity rather than a focus on GM per se. Response to concerns about GM food (64\%) was similar to those for food coloring (67\%), pesticides (70\%), food preservatives $(69 \%)$ and food additives $(71 \%)$.
Communication with consumers is critical and is needed to overcome misperceptions by $15 \%$ of consumers that GM fruit and vegetables are common in the Australian food chain ${ }^{[3]}$, when in fact none is GM. Even the best of intentioned strategies can have the perverse effect by increasing consumer concerns. However, concern may not denote opposition, but rather acknowledgment that it was an important consideration to the consumer.

\section{Trust}

Trust in the food supply chain is critical to support both domestic and imported food. Australian consumers generally trust local food safety and in a recent survey, $81 \%$ of people felt that food products sourced from Australia, the UK and the USA were safe to eat. This contrasted with $66 \%$ who did not feel confident with the safety of food products imported from Asia ${ }^{[4]}$.

We are fortunate that Australia has an enviable reputation for food safety, producing clean and green food. We should build on trust in these attributes (though they are not well defined) by continued high-level performance and objective evidence of adherence to global standards. Reputation is all important. It is extremely difficult to regain consumer confidence once it is tarnished (as shown in recent food scandals).

More generally, a number of actions are needed to build and maintain trust.

- Build confidence in the regulatory system by ensuring accountability and transparency. This regulatory system also needs to be responsive to both consumer and business issues. It depends on a collaborative effort between government and industry.

- Support mechanisms to build confidence in food integrity through traceability mechanisms and labeling requirements. These allow food contamination outbreaks to be localized to specific elements of the food chain, rather than all parts of the chain being held responsible.

- Develop an evidence-based green index/labeling to facilitate an environmental cost calculation and potentially lead to the development of environmental credit to reward more sustainable producers.

- Acknowledge the role of international private industry food standards, particularly in maintaining the integrity of food supply chains in regional areas, developing countries and commercial networks.

- Encourage the harmonization of government and private food standards wherever possible. This will improve food safety outcomes and reduce supply chain costs leading to a more sustainable compliance system with greater integrity.

- Provide choice for consumers allowing the fast adopters of technology to lead the way with new 
technology while other consumers take a more References measured pace.

\section{Final comment}

Consideration of the pyramid of interlocking issues systems, technology, culture and trust - will facilitate the delivery of food safety now and in the future.

Compliance with ethics guidelines Kaye Basford, Richard Bennett, Joanne Daly, Mary Ann Augustin, Snow Barlow, Tony Gregson, Alice Lee, Deli Chen, and Matt Wenham declare that they have no conflict of interest or financial conflicts to disclose.

This article does not contain any studies with human or animal subjects performed by any of the authors.

1. Miles S, Brennan M, Kuznesof S, Ness M, Ritson C, Frewer L J. Public worry about specific food safety issues. British Food Journal, 2004, 106(1): 9-22

2. Mohr P, Golley S. Responses to GM food content in context with food integrity issues: results from Australian population surveys. New Biotechnology, 2016, 33(1): 91-98

3. Ipsos. Community attitudes towards emerging technology issues - biotechnology. Report of Findings for the Department of Industry, Innovation, Science, Research and Tertiary Education. Paris: Ipsos Social Research Institute, 2013, 135

4. Lockie S, Pietsch J. Public opinion on food security. Available at Australian National University Website on September 21, 2016 